\title{
HERMES In Retrospect: An Experiment Based On Innovative Technologies
}

\author{
Erhard Steffens \\ Physikalisches Institut, University of Erlangen-Nürnberg, \\ Erwin-Rommel-Str. 1, D-91058 Erlangen, Germany; \\ and \\ HERMES Collaboration, DESY (Hamburg).
}

\begin{abstract}
Proposed in 1990 the HERMES experiment aimed at the measurement of spindependent observables in deep-inelastic scattering of electrons on few-nucleon targets. It relied on the availability of polarized stored electrons and on gas targets of sufficient density and polarization. Data were taken from 1995 to 2007. This paper describes how these key techniques were developed and applied during the run.
\end{abstract}

Keywords: Polarized deep-inelastic scattering, polarized electron storage ring, polarized hydrogen and deuterium gas targets, spin structure of the nucleon.

PACS: 29.25.Pj, 29.27.Hj, 24.70.+s, 25.30.Dh

\section{PROLOGUE: HERA 1990-2007}

The 'Hadron-Elektron Ring-Anlage' HERA was designed as proton-electron collider with unprecedented resolution and built in the $2^{\text {nd }}$ half of the 1980 's. First collisions were observed in 1990. The layout of the electron ring allowed for longitudinal electron polarization at the experiments. Two collider experiments (H1 [1] and ZEUS [2]), and two fixed-target experiments (HERMES [3] and HERA-B [4]) have been operational. In summer 2007, the running of the HERA complex was terminated. The review talks of HERA and the various experiments can be found at the HERA-Fest homepage [5]. An aerial view of HERA which is located north-west of the City of Hamburg is shown in Fig.1.

\section{INTRODUCTION}

Deep-inelastic lepton scattering (DIS) is a powerful method to study the internal structure of the nucleon. Using multi-GeV beams of electrons (e.g. at SLAC) or muons (e.g. at CERN), the structure functions $F_{1}$ and $F_{2}$ have been measured, reflecting the momentum distribution of the quarks. At the end of the 1980's a new question arose: How is the spin of the nucleon carried by the quarks, or more generally by the constituents? Here new methods were required. The first result was published by EMC in 1988 [6], indicating that a low fraction of the nucleon spin only is carried by quarks. The experiment was performed using a muon beam at CERN which was 


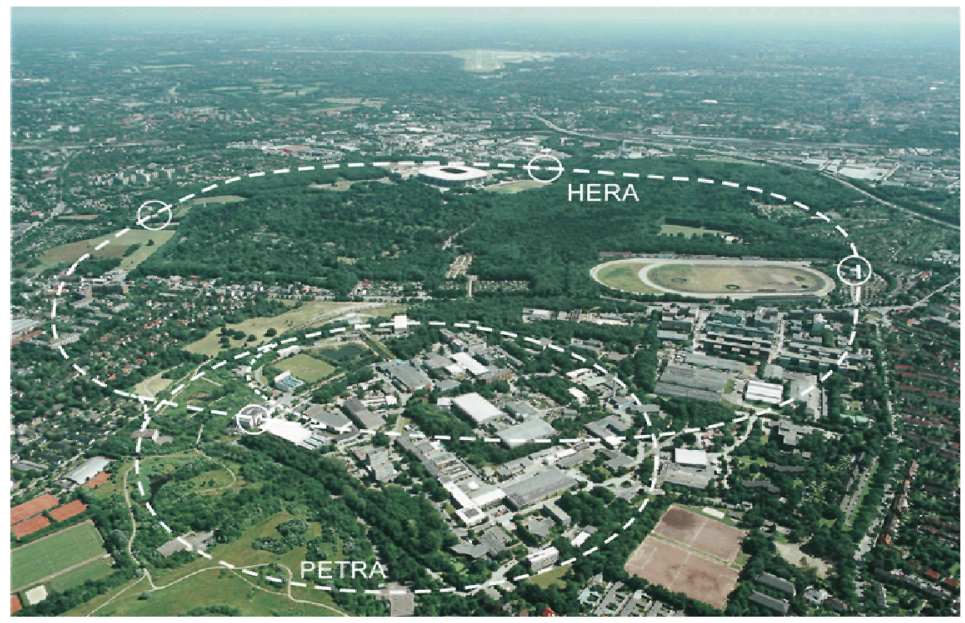

FIGURE 1 Aerial view of the HERA complex with the injector ring PETRA-II and the DESY site in the foreground. The experimental halls are marked by circles, H1 and ZEUS left and right, resp., and HERMES in the back. The airport is visible in the background.

produced by the parity-violating decay of pions in flight leading to polarized muons.

Why polarized DIS is sensitive to quark polarization? This can be explained in terms of the conserved helicity sum of the particles involved:

- The lepton with helicity $+1 / 2$ emits a virtual photon with helicity +1 while flipping its own helicity to $-1 / 2$;

- Quarks with helicity $-1 / 2$ only can absorbe helicity +1 photons while flipping their helicity to $+1 / 2$.

In total, leptons with helicity 'plus' scatter on quarks with helicity 'minus' only, and vice versa. By varying the helicity of the nucleons, i.e. the longitudinal polarization of the target, the quark polarization with respect to the nucleon spin direction can be measured, expressed as $\Delta \mathrm{q}=\mathrm{q}^{+}-\mathrm{q}^{-}, \mathrm{q}^{\mathrm{h}}$ being quark distribution functions for different helicities $h$. From inclusive measurements of the scattered lepton, the spin structure function $\mathrm{g}_{1}(\mathrm{x})$ can be derived. Using semi-inclusive DIS the quark polarizations $\Delta \mathrm{q}$ can be determined for individual quark flavors $q=u, d, s$. For an overview of the HERMES results see [7].

\section{A New Experimental Approach: Intense Polarized Beams Stored in a Ring Interacting with Polarized Gas Targets}

At the end of the 1980's several ideas were around how such a new concept could be realized. Polarized few nucleon targets like hydrogen, deuterium or ${ }^{3} \mathrm{He}$ for the measurement of spin-dependent observables have distinct advantages over solid polarized targets, like high purity and low background. In order to reach the 'benchmark density' $\mathrm{t}=10^{14}$ atoms $/ \mathrm{cm}^{2}$ defined 20 years ago [8], the application of a Storage Cell is mandatory which has been proposed in 1966 by W. Haeberli (for an 
overview of the storage cell technique see [9]). Such densities for hydrogen have been demonstrated for the first time in 1992 at the Heidelberg TSR [10].

Polarized stored beams of high energy can be produced by injecting polarized beams of low energy and accelerating them to the desired energy. In a circular machine, e.g. a synchrotron, this means crossing of many depolarizing resonances. For ultra-relativistic electrons another approach exists: Polarizing a beam at the final energy by 'Radiative Polarization' proposed by Sokolov and Ternov in 1964 [11]. This technique has been studied successfully at various laboratories [12]. The remaining question was whether longitudinal electron polarization of sufficient degree can be produced in a high energy storage ring like HERA.

In the following we discuss how these basic techniques have been developed and applied for the HERMES experiment [3].

\section{POLARIZED ELECTRON BEAMS STORED IN HERA}

In an ideal machine radiative polarization $\mathrm{P}$ builds up to a maximum value $\mathrm{P}_{0}=$ $8 / 5 \sqrt{3}=0.924[11,12]$. The time constant for the polarization build-up of electrons is given by ( $\rho=$ dipole bending radius, $R=C / 2 \pi, C=$ machine circumference)

$$
\tau_{0}(\mathrm{sec})=98.66 \frac{[\rho(m)]^{\beta}}{[E(G e V)]^{5}} \frac{R}{\rho}
$$

resulting in about $39 \mathrm{~min}$ for the nominal energy of the HERA electron ring of $\mathrm{E}=$ $27.5 \mathrm{GeV}$. The effect is basically due to the huge $\gamma^{5}$ factor with which the transverse bending field transforms into the electron rest frame resulting in spontaneous spin flips by $\mathrm{M} 1$ dipole transitions [13]. In order to achieve high $\mathrm{P}$, the depolarization of the ring has to be minimized e.g. by proper choice of the spin tune

$$
v_{s}=\gamma \frac{g-2}{2}=\gamma a
$$

$\left(\gamma=\right.$ Lorentz factor, $\mathrm{g}=\mathrm{g}$-factor of the electron, $\mathrm{a}=$ electron anomaly $\approx 1.160 \cdot 10^{-3}$ ) to a half-integer tune of 62.5 at $27.5 \mathrm{GeV}$, by 'spin matching' and and by performing 'harmonic orbit corrections' [12].

For longitudinal polarization at the interaction point (IP) a pair of Spin Rotators is required which turns the spin from the stable vertical direction, i.e. parallel to the bending field, into the longitudinal and then back into the vertical direction. For deflection by a transverse B-field, the spin precession angle is given by $\alpha_{\mathrm{p}}=\alpha_{\text {orbit }} \cdot v_{\mathrm{s}}$, i.e. huge compared with the deflection angle $\alpha_{\text {orbit. }}$ Therefore spin and orbit deflection do not commute, and with a set of dipoles the spin can be rotated without net deflection. The HERA 'Mini Rotator' [14] is sketched in Fig. 2. Each rotator is about $60 \mathrm{~m}$ long. The maximum orbit distortion is $\pm 22 \mathrm{~cm}$, which requires to move the dipoles by means of motor-driven supports accordingly.

The polarization of the stored electrons is measured via Compton scattering using circular polarized photons. The backscattered gammas are detected by a calorimeter. 


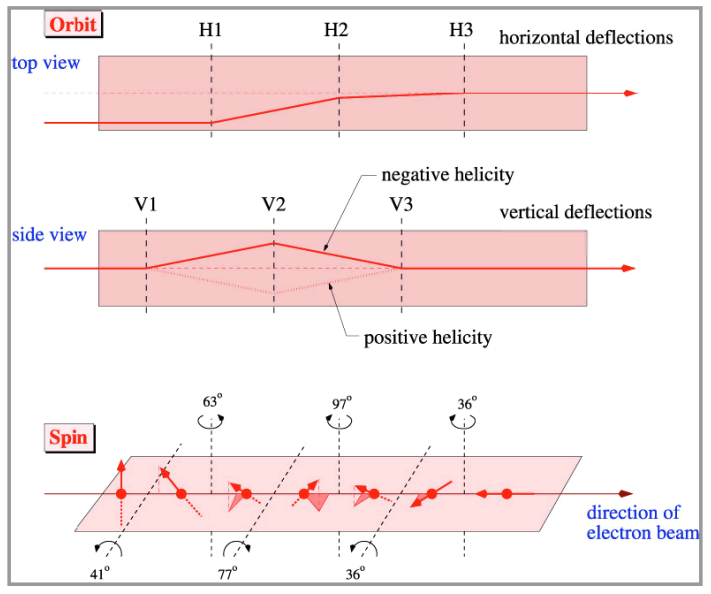

FIGURE 2 Sketch of the HERA Mini Rotator $[14,15]$. The orbit deflection is shown as top and side view, whereas the corresponding spin precession angles are indicated at the bottom. As a net result, the spin is turned from 'up' into 'backwards'.

The transverse polarimeter (TPOL) in the West hall detects the tiny up-down asymmetries associated with vertical polarization, while the LPOL in the East hall close to the HERMES IP utilizes the count rate asymmetry caused by longitudinal polarization.

Longitudinal polarization has been observed for the first time at HERA in 1994 [15]. The maximum polarization ever observed at HERA was about $70 \%$, and in the average around 55\% has been detected over longer runs. During HERA-run II, the beam polarization was reduced due to enhanced beam-beam interaction and two additional pairs of spin rotators for ZEUS and $\mathrm{H} 1$.

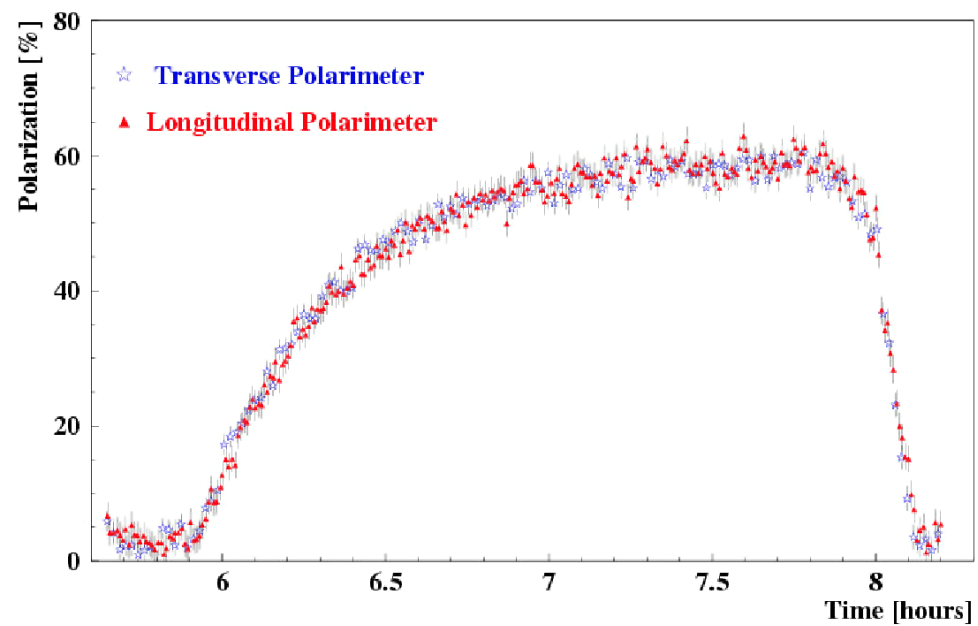

FIGURE 3 Comparison of TPOL $(*)$ and LPOL $(\Delta)$ measurements during polarization rise after the beam has been depolarized artificially using an rf coil (HERMES polarimeter group 1997), 
In Fig.3, a comparison of the two polarimeters is shown. By fitting with a function $\mathrm{P}(\mathrm{t})=\mathrm{P}_{0}\left[1-\exp \left(\mathrm{t} / \tau_{\text {eff }}\right)\right]$ an effective polarization rise time $\tau_{\text {eff }}$ can be determined. From the ideal rise time $\tau_{0}$ (see equ. 2), the saturation value of the polarization in a real machine can be calculated using $\mathrm{P}_{\infty}=\left(\tau_{\mathrm{eff}} / \tau_{0}\right) \cdot \mathrm{P}_{0}=0.924\left(\tau_{\mathrm{eff}} / \tau_{0}\right)$. In this way, the polarimeters could be calibrated. The absolute error is around $3 \%$.

\section{POLARIZED GAS TARGETS}

As already mentioned, a storage cell is employed in order to obtain high density [9]. The basic idea is depicted in Fig.4. A polarized atomic beam from a source is injected into the feed tube of the T-shaped cell, resulting in a pressure rise at the center. The beam traverses the straight beam tube with typical length of about $40 \mathrm{~cm}$. Scattered particles penetrate the thin cell walls and are detected e.g. left and right with respect to the beamdirection. The gain in density of this extended target compared with the atomic beam without cell is two orders of magnitude.

Atoms trapped in the cell perform a high number of wall collisions before escaping, usually in the order of several hundred. Therefore a suitable coating is required in order to prevent depolarization and recombination of the stored atoms. Teflon, the standard coating for the storage bulb of the hydrogen Maser [16] does not withstand X-rays present in electron storage rings. In 1988 an Argonne-Novosibirsk collaboration found that a silicone ('Dryfilm') coated Al storage cell in the VEPP-3 electron storage ring at BINP (Novosibirsk), fed with polarized deuterium atoms, conserved nuclear tensor polarization to a high degree over several months [17]. Therefore, Dryfilm had been foreseen as coating for the HERMES target cell.

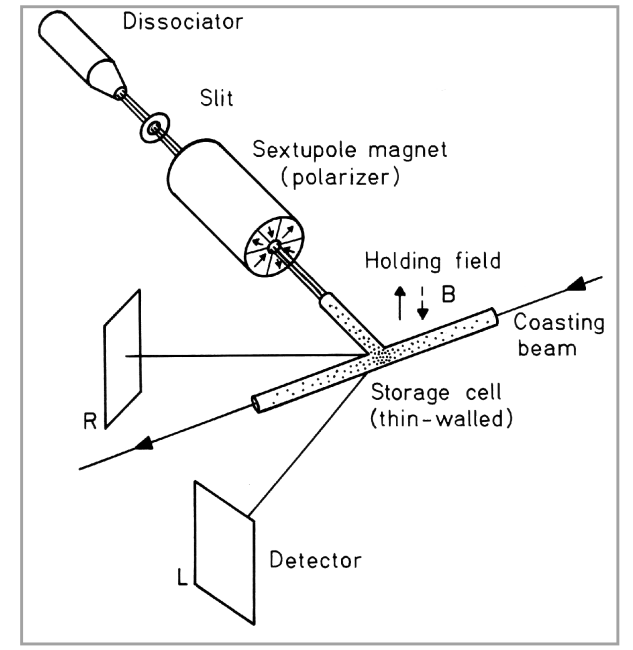

FIGURE 4 Principle of the storage cell target [9, 10]. 
The first test of a storage cell target for hydrogen was performed at the test storage ring TSR (MPI Heidelberg) in $1992[18,10]$. The basic arrangement was as shown schematically in Fig.4. A weak vertical holding field was applied along the cell. The ABS had two groups of sextupole magnets and a if transition in between for the selection of a single substate, the 'pure' upper state $\mid 1>$ with full nuclear polarization +1 at low field ${ }^{1}$. For increasing the density the cell could be cooled down to $50 \mathrm{~K}$. The measured polarization showed that about $90 \%$ of the injected polarization is conserved at temperatures down to $80 \mathrm{~K}$, where a density of $1.0 \cdot 10^{14} / \mathrm{cm}^{2}$ was found for two substates. - A similar test of a cryogenic optically pumped polarized ${ }^{3} \mathrm{He}$ target was performed at the IUCF Cooler ring in 1992 [19]. A density of $1.5 \cdot 10^{14} / \mathrm{cm}^{2}$ and a polarization of $50 \%$ had been measured. These results constituted important milestones for the final approval of HERMES which happened in July 1993.

\section{The ${ }^{3}$ He Target}

${ }^{3} \mathrm{He}$ is a spin- $1 / 2$ nucleus. As the two protons are in good approximation spinsaturated, polarized ${ }^{3} \mathrm{He}$ basically constitutes a polarized neutron target. Due to its noble gas configuration there is very little depolarization by the walls down to temperatures as low as 10-15 K [20]. In order to prevent 'motional' depolarization a large-volume homogenous guide field is required. The target gas was polarized using the metastability-exchange optical-pumping technique and injected into a cold target cell of thin walled pure Al sheets. Optical pumping was performed by infrared light of circular polarization in a quartz cell. Polarized gas flowed via the exit capillary into the target cell. A system of tungsten collimators protected the cell against the synchrotron radiation background emerging from the machine. For more details see [20].

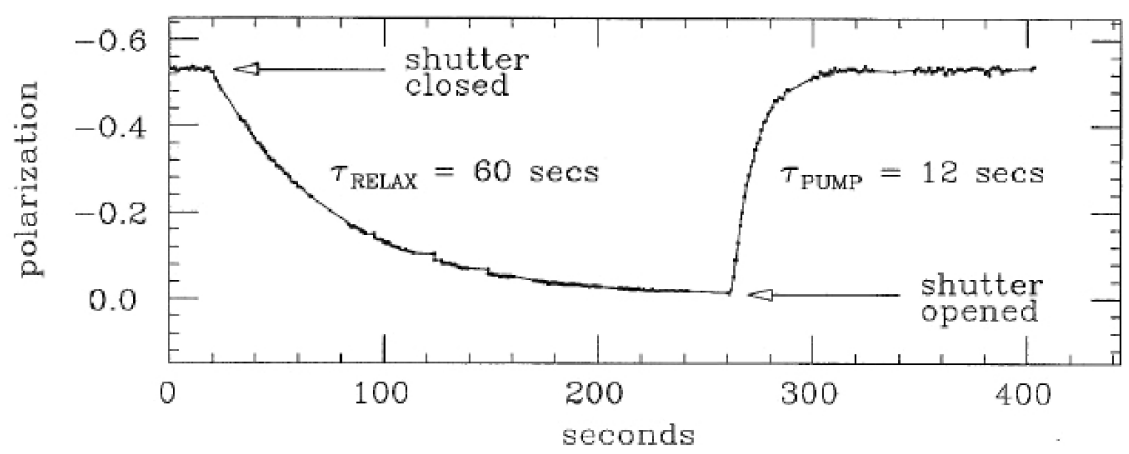

FIGURE 5 Polarization for the ${ }^{3} \mathrm{He}$ target in flow mode as function of time. The laser light is controlled by means of a shutter. The exponential changes of the polarization in the quartz cell due to pumping and relaxation are clearly visible. The latter process also includes dilution by fresh unpolarized gas. [20].

\footnotetext{
1 The hfs states of hydrogen and deuterium are labelled by $|1\rangle-|4\rangle$ and $|1\rangle-16>$, resp., with decreasing energy in a B-field [9, 24].
} 
The ${ }^{3} \mathrm{He}$ target was used in the first HERMES run in 1995 to measure $\mathrm{g}_{1}(\mathrm{x})$ of the neutron [21], the first physics result of HERMES. In routine operation, the polarization was between 40 and 54\% (average during data taking 46\%) at a thickness of $1.0 \cdot 10^{15} / \mathrm{cm}^{2}$ given by the maximum allowed contribution to the beam life time. The experiment was rolled into beam position on February 6, 1995 (see Fig. 6).

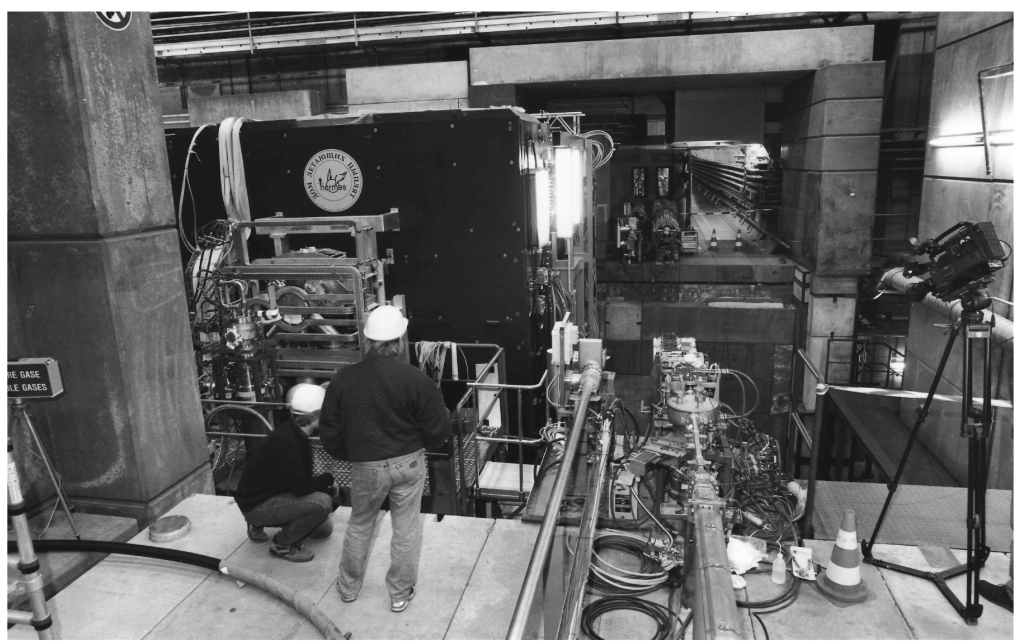

FIGURE 6 Moving the experiment into beam position on February 6, 1995. The electron and proton beam lines are visible in the front. The tunnel is seen in the back. The platform with spectrometer magnet and He-target moves from left to right.

\section{The Hydrogen and Deuterium Target}

Apart from demonstrating the key parameters density and polarization there were many more open questions like:

1. Wake field heating of the narrow cell

2. Effect of synchrotron radiation on coating and background

3. Bunch field depolarization of the target gas

4. How to perform precise target polarimetry to $3 \%$ in precision

In 1994, a test experiment at the final location in the East hall was conducted, comprising the system of the movable $\mathrm{C} 1$ and the fixed $\mathrm{C} 2$ collimators - the latter having a free opening of $6 \mathrm{~mm}$ in height (!) - a prototype target chamber with Dryfilm coated cell, and a set of prototype detectors to study the background conditions. It could be demonstrated that closing of the (upstream) $\mathrm{C} 1$ collimator to the design opening suppressed the synchrotron radiation background close to the beam tube quantitatively. This gave confidence that items $(1,2)$ were under control. Analysis of the rf depolarization mechanism [22] lead to the conclusion that depolarization occurs at distinct 'resonant' B-fields the density of which decreases with field strength. There fore, a guide field of about $0.34 \mathrm{~T}$ in strength has been chosen as working point [23]. As a precise beam-independent method a sampling polarimeter (BRP) has been developed, analyzing a tiny beam extracted from the center of the target cell. The BRP 
was able to measure the substate populations $n_{i}(i=1-4$ for hydrogen, and $i=1-6$ for deuterium) with $1 \%$ precision in a couple of minutes. For an overview of the HERMES hydrogen and deuterium target and references to specific components see [24].

\section{Longitudinal Target Polarization}

The H\&D target is shown in Fig. 7. The polarized atomic beam is produced by means of an Atomic Beam Source (ABS, right) and injected into the storage cell located on the axis of the $27.5 \mathrm{GeV}$ electron beam in a longitudinal guide field of about $0.34 \mathrm{~T}$. A sample beam extracted from the cell is analyzed by the BRP polarimeter (left). In the back, the Vertex and Front drift chambers in front of the spectrometer magnet are visible. In the foreground (left) the proton beam line is shown, passing the experiment virtually without interaction of the $920 \mathrm{GeV}$ protons.

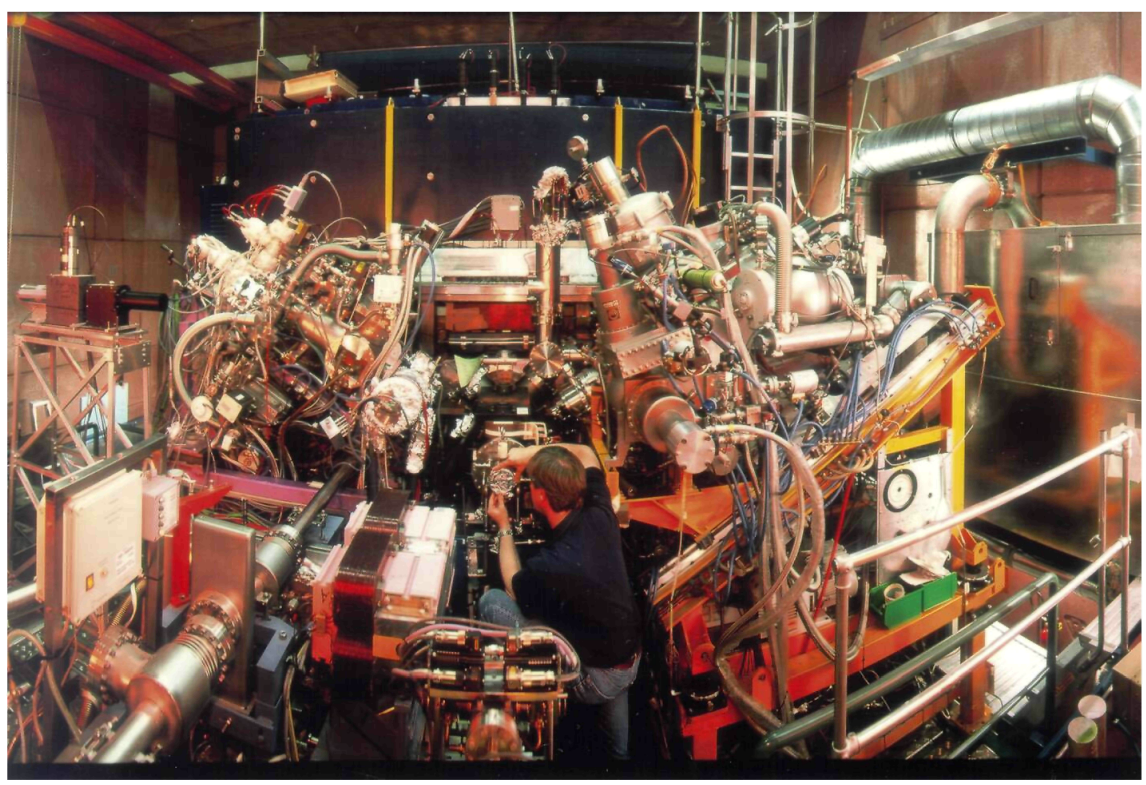

FIGURE 7 The hydrogen and deuterium target during HERA run I with longitudinal target polarization (for details see text).

The longitudinal 'strong' guide field has two important advantages:

1. No synchrotron radiation induced by the guide field,

2. Perpendicular orientation of guide field and the closed magnetic field lines of the bunch field.

(2) was important for the transitions between hfs levels of the target gas induced by the beam rf. Perpendicular orientation corresponds to transitions with $\Delta \mathrm{m}_{\mathrm{F}}= \pm 1$, called ' $\pi$-transitions'. As a consequence, in a strong field there is ample space between neighboring resonances, as demonstrated by the measurement of the $\pi$-resonances induced by the HERA electron beam, shown in Fig. 8. With proper choice of the working point rf depolarization can be completely suppressed. - In Tab. 1, typical 
results of the determination of the target polarization are presented. The result measured by the BRP has been corrected for various effects listed in the table, like depolarization due to recombination, wall collisions and spin exchange collisions. The error during the run in 2000 with longitudinally polarized deuterium is particularly low. This is partly due to the low magnetic moment of the deuteron which reduces several error contributions practically to zero.

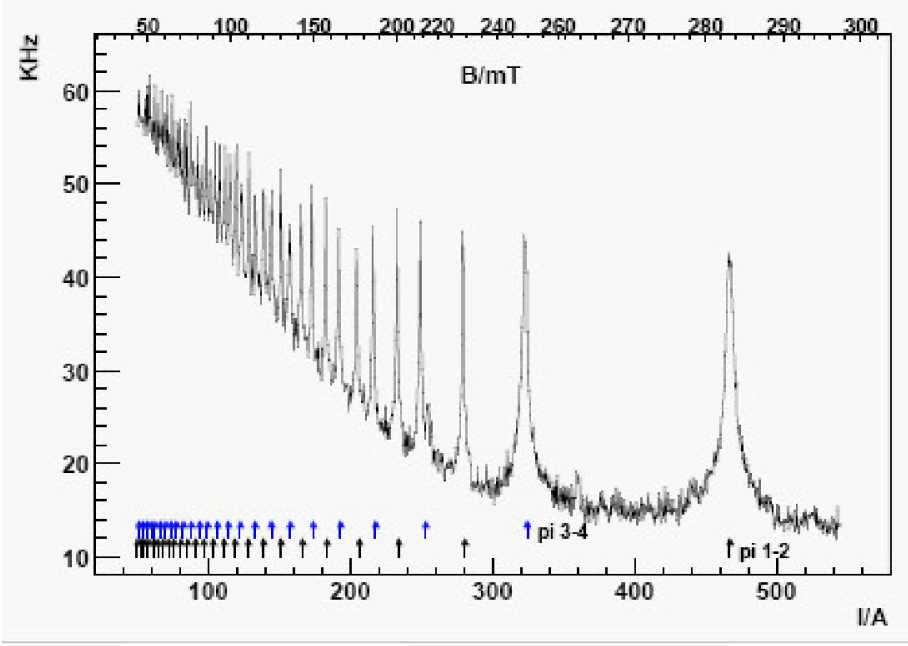

FIGURE 8 Depolarizing resonances for hydrogen induced by the HERA electron beam [25], as function of magnet current (bottom) and field strength (top). Hydrogen states $|1\rangle$ and $|4\rangle$ are injected into the target cell, and states $|2\rangle$ and $|3\rangle$ detected by the BRP. The signal is given as BRP particle count rate in $\mathrm{kHz}$. The expected location of the pi 1-2 and pi 3-4 resonances are indicated by arrows. The rise of the signal at low field is predominantly due to spin exchange collisions between atoms in different hfs states.

\section{Transverse Target Polarization}

During the long shutdown 2000/01, a target magnet with highly uniform vertical guide field was installed $[24,26]$. Here due to the orientation of static and rf B-fields a new class of densely spaced depolarizing resonances appeared, the $\sigma$-resonances with $\Delta \mathrm{m}_{\mathrm{F}}=0$. The spacing at high field was about $0.4 \mathrm{mT}$. Deflection of the electron beam had to be compensated by a 'dog leg' type chicane consisting of three dipoles one being the target magnet. Because of the significant level of synchrotron radiation produced in the target of the order of $1-2 \mathrm{~kW}$ in power, the B-field was limited to 0.30 $\mathrm{T}$, resulting in slightly lower target polarization (see Tab. 1, last column) compared with the longitudinal target field of about $0.335 \mathrm{~T}$.

In order to further reduce the fairly weak contribution of the $\sigma$-resonances to depolarization, an extra coil with two windings was installed along the cell close to the center. By tuning its current the homogeneity, predominantly in horizontal direction, 


\begin{tabular}{|c|c|c|c|}
\hline Target/year & $\mathbf{H} \|(\mathbf{1 9 9 7})$ & $\mathbf{D} \|(\mathbf{2 0 0 0})$ & $\mathbf{H} \perp \mathbf{( 2 0 0 3 / 0 4 )}$ \\
\hline $\mathrm{P}_{\mathrm{t}}$ & $0.851 \pm 0.033$ & $0.845 \pm 0.028$ & $0.786 \pm 0.036$ \\
\hline$\Delta \alpha_{\mathrm{r}}$ & 0.055 & 0.003 (absent) & absent \\
\hline$\Delta \mathrm{P}_{\mathrm{SE}}$ & 0.035 & $\leq 0.001$ (absent) & 0.055 \\
\hline$\Delta \mathrm{P}_{\mathrm{WD}}$ & 0.02 & $\leq 0.01$ (absent) & 0.055 \\
\hline$\Delta \mathrm{P}_{\mathrm{BI}}$ & - & - & $\leq 0.01$ \\
\hline $\mathrm{t}\left(10^{14} \mathrm{nucl} / \mathrm{cm}^{2}\right)$ & 0.7 & 2.1 & 1.1 \\
\hline $\mathrm{FOM}\left(\mathrm{P}^{2} \mathrm{t}\right)$ & 0.5 & 1.5 & 0.7 \\
\hline
\end{tabular}

TABLE 1. Target polarization with total error, for the various running periods of the HERMES target (Hermes target group, and [24]). The first two runs were done with longitudinal, the last with vertical or transverse spin. Fractional errors $\Delta \ldots$ are given for the different effects contributing to the total uncertainty $(\alpha=$ dissociation fraction, $\mathrm{r}=$ recombination, $\mathrm{SE}=$ spin exchange, $\mathrm{WD}=$ wall depolarization, $\mathrm{BI}=$ beam induced depolarization). The values given in the table are averages over distinct periods indicated in brackets. The 'longitudinal hydrogen' run took place in 1996 \& 97, the 'longitudinal deuterium' run was in 1998-2000, and the 'transverse hydrogen' run was performed during 2002-2005. In the last line, the relative 'figure of merit' is given.

could be improved substantially. A scan of the $\sigma$-resonances in a small region in the vicinity of the working point is shown in Fig. 9. The spacing between the two resonances is smaller than their width. The relative change off/on resonance is about $2 \%$. The remaining depolarization could be determined unambiguously only by dumping an intense beam, which obviously could only be performed a few times. The results indicate small depolarization also in between resonances [25].

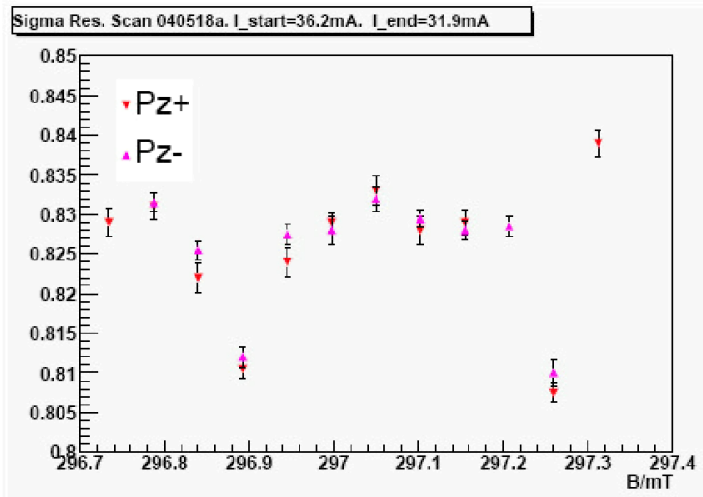

FIGURE 9 Scan of the target polarization as function of the holding field $\mathrm{B}$ in $\mathrm{mT}$ [25]. The dips at about 296.89 and 297.26 $\mathrm{mT}$ correspond to two $\boldsymbol{\sigma}$ resonances. The injected vector polarization $(+/-)$ is indicated. There is no significant difference between the two signs.

\section{CONCLUSIONS}

HERMES is a good example of how new experimental techniques enable the measurement of novel observables not accessible before. Stored beams of polarized electrons and positrons, in conjunction with polarized targets of light nuclei and with an excellent spectrometer, have led to many exciting new results on spin structure of the nucleon $[7,28]$. At the end of HERA running these techniques are at a high level and ready to be utilized for new challenging experiments. 


\section{ACKNOWLEDGMENTS}

I gratefully acknowledge the work of numerous HERMES and DESY colleagues and students who made the HERMES experiment a pleasant adventure and a big success. Special thanks are due to the funding agencies, in particular the BMBF and INFN, the DESY management for continuous support, and to the members of the target group for their dedication and indefatigable effort. This work is partly supported by BMBF grant 06ER144.

\section{REFERENCES}

1. http://www-h1,desy,de

2. http://www-zeus.desy.de/

3. http://www-hermes.desy.de/

4. http://www-hera-b.desy.de/

5. http://hera-fest.desy.de/

6. J. Ashman et al, Nucl. Phys. B328 (1989) 1.

7. K. Rith, Progr. Part. Nucl. Phys, 49 (2002) 245.

8. E. Steffens et al, in: Proc. 3rd LEAR Workshop, Tignes 1985, U. Gastaldi et al (Eds.), Editions Frontieres, p. 245.

9. E. Steffens and W. Haeberli, Rep. Progr. Phys, 66 (2003) 1887.

10. K. Zapfe et al, Rev. Sci. Instrum. 66 (1995) 28.

11. A.A. Sokolov and I.M. Ternov, Sov.Phys. Doklady 8 (1964) 1203.

12. D.P. Barber, in: Proc. Spin 1998, Protvino, N.E. Tyurin et al (Eds.), World Scientific (1999) p. 246.

13. J.D. Jackson, Rev. Mod. Phys. 48 (1976) 417.

14. K. Steffen, in : Proc. High Energy Spin Physics, Minneapolis 1988, K.J. Heller (Ed.), AIP Conf. Proc. No. 187 (1989), p. 919.

15. D.P. Barber et al, Phys. Lett. 343B (1995) 436.

16. H.M. Goldenberg, D. Kleppner and N.F. Ramsey, Phys. Rev. Lett. 5 (1960), and Phys. Rev. 126 (1962) 603.

17. D.K. Toporkov et al, in : Proc. High Energy Spin Physics, Minneapolis 1988, K.J. Heller (Ed.), AIP Conf. Proc. No. 187 (1989), p.1286, see also: HERMES Collaboration: Technical Design Report, DESY-PRC 93/06 (1993), Fig. 2.4 .3 on p.II-26.

18. W. Haeberli, in: Proc. High Energy Spin Physics, Nagoya 1992, T. Hasegawa et al (Eds.), Universal Academy Press, Tokyo (1993), p. 335.

19. K. Lee et al, Phys. Rev. C29 (1994) 2001.

20. D. DeSchepper et al, Nucl. Instr. Meth. A419 (1998) 16.

21. K. Ackerstaff et al, Phys. Lett. B404 (1997) 383.

22. HERMES Collaboration: Technical Design Report, DESY-PRC 93/06 (1993).

23. K. Ackerstaff et al, Phys. Rev. Lett. 82 (1999) 1164.

24. A. Airapetian et al, Nucl. Instr. Meth. A540 (2005) 68.

25. P. Tait: Beam-Induced Depolarization at the HERMES Transversily Polarized Hydrogen Target. Dissertation, Univ. of Erlangen-Nürnberg (2006); http://www-hermes.desy.de/notes/prot/docfull_thesis-index.html

26. D. Reggiani: Precision Polarization Measurement of the HERMES H/D Gas Target. Dissertation, Univ. of Ferrara (2003); http://www-hermes.desy.de/notes/prot/doc-full_thesis-index.html

27. S. Wang: Improvements of the HERMES Transverse Hydrogen Target and Calculations of the Sextupole Magnet. Dissertation, Univ. of Peking (2004); http://www-hermes.desy.de/notes/prot/docfull_thesis-index.html

28. D. Hasch, in: Proc. $17^{\text {th }}$ Int. Spin Physics Symp., Kyoto 2006, K. Imai et al (Edts), AIP Conf. Proc. No. 915, New York (2007) 307. 